\title{
Peroxisome proliferator-activated receptor- $\gamma$ coactivator-1 and insulin resistance: acute effect of fatty acids
}

\author{
J. Hoeks • M. K. C. Hesselink • A. P. Russell • \\ M. Mensink • W. H. M. Saris • R. P. Mensink • \\ P. Schrauwen
}

Received: 24 March 2006 / Accepted: 25 May 2006 / Published online: 1 August 2006

(C) Springer-Verlag 2006

\begin{abstract}
Aims/hypothesis Peroxisome proliferator-activated receptor (PPAR)- $\gamma$ coactivator-1 (PPARGC1), a coactivator regulating the transcription of genes involved in oxidative metabolism, is downregulated in patients with type 2 diabetes and in their first-degree relatives. Whether this downregulation is a cause or effect of early aberrations in the development of insulin resistance, such as disturbances in fat metabolism, is unknown. We examined whether lipidinduced insulin resistance was associated with downregulation of expression of skeletal muscle genes involved in oxidative metabolism and mitochondrial biogenesis in humans.

Materials and methods Nine healthy lean male subjects underwent a 6-h hyperinsulinaemic-euglycaemic clamp with simultaneous infusion of either a lipid emulsion or glycerol as a control. Blood was sampled at regular time points and muscle biopsies were taken before and after every test. Intramuscular triacylglycerol (IMTG) content
\end{abstract}

J. Hoeks $(\bowtie) \cdot$ M. Mensink $\cdot$ W. H. M. Saris $\cdot$ R. P. Mensink

P. Schrauwen

Department of Human Biology, Nutrition and Toxicology

Research Institute Maastricht (NUTRIM), Maastricht University,

P.O. Box 616, 6200 MD Maastricht, The Netherlands

e-mail: j.hoeks@hb.unimaas.nl

J. Hoeks • M. K. C. Hesselink • R. P. Mensink - P. Schrauwen

Wageningen Centre for Food Sciences (WCFS),

Wageningen, The Netherlands

M. K. C. Hesselink

Department of Movement Sciences, Nutrition and Toxicology

Research Institute Maastricht (NUTRIM), Maastricht University,

Maastricht, The Netherlands

A. P. Russell

Clinique Romande de Readaptation SUVA Care,

Sion, Switzerland was determined by Oil Red O staining and gene expression was measured by quantitative PCR.

Results Lipid infusion resulted in a 2 2.7-fold increase in plasma NEFA levels and a $31 \pm 6 \%$ decrease in insulin sensitivity $(p=0.001)$. The infusion of lipids resulted in a $\sim 1.6$-fold increase in IMTG ( $p=0.02)$, whereas during the clamp with glycerol infusion IMTG tended to decrease to $\sim 53 \%$ of preinfusion levels $(p=0.065)$. Lipid infusion decreased PPARGC1A, PPARGC1B and PPARA expression to $\sim 61,77$ and $\sim 52 \%$ of basal values respectively, whereas expression of uncoupling protein 3 was upregulated 1.8fold (all $p<0.05$ ).

Conclusions/interpretation Acute elevation of plasma NEFA levels, leading to muscular fat accumulation and insulin resistance, downregulates PPARGC1A, PPARGC1B and PPARA expression, suggesting that the decrease in PPARGC1 expression observed in the (pre)diabetic state may be the result, rather than the cause of lipid-induced insulin resistance.

Keywords Fatty acids · Insulin resistance .

Lipid metabolism · PGC1 - Type 2 diabetes mellitus
Abbreviations
ACACB
acetyl-coenzyme A carboxylase $\beta$
COX4I1 cytochrome $\mathrm{c}$ oxidase subunit IV isoform 1
CPT1B carnitine palmitoyltransferase 1B (muscle)
IMTG
NRF intramuscular triacylglycerols
PPARA nuclear respiratory factor
PPARD peroxisome proliferator-activated receptor $\alpha$ PPARGC1A peroxisome proliferator-activated receptor- $\gamma$ coactivator $-1 \alpha$ 
PPARGC1B peroxisome proliferator-activated receptor- $\gamma$ coactivator- $1 \beta$

$\mathrm{UCP} 3$

SOD2

uncoupling protein 3

superoxide dismutase 2, mitochondrial

\section{Introduction}

Accumulation of triacylglycerols in skeletal muscle is closely associated with insulin resistance [1] and the insulin-resistant state can even be induced in healthy individuals by acute infusion of lipids, probably as a result of accumulation of lipid derivatives and subsequent disturbance of insulin signalling [2]. Recently, impaired mitochondrial functioning has also been identified as a key determinant in the aetiology of type 2 diabetes. Thus, it has been shown that mitochondrial oxidative phosphorylation is reduced in insulin-resistant subjects [3] and that genes involved in mitochondrial oxidative metabolism are coordinately downregulated in type 2 diabetes [4, 5].

Many of these oxidative genes are under the transcriptional control of peroxisome proliferator-activated receptor $\gamma$ coactivator-1 $\alpha$ (PPARGC1A, also known as PGC- $1 \alpha$ ) which stimulates oxidative phosphorylation, mitochondrial biogenesis and the generation of oxidative type 1 muscle fibres [6]. In addition to the downregulation of the so-called OXPHOS gene set, it has been shown that the expression of PPARGC1A itself is also reduced in type 2 diabetic patients $[4,5]$. Strikingly, the same observations have been reported in non-diabetic subjects with a family history of diabetes [5].

Peroxisome proliferator-activated receptor- $\gamma$ coactivator$1 \beta$ (PPARGC1B) (also known as PGC-1 $\beta$ ), a structural homologue of PPARGC1A, displays a tissue distribution similar to that of PPARGC1A and influences a similar set of oxidative target genes in both muscle and liver cells [7, 8]. Thus, overexpression of PPARGC1B in L6 myoblasts increased mitochondrial content and oxygen consumption [9] and mice overexpressing PPARGC1B showed increased energy expenditure and were protected against diet-induced obesity [10]. However, besides the existing functional overlap between the two PPARGC1 homologues, the transcription of PPARGC1A and PPARGC1B seems to be differentially regulated. Hence, unlike the expression of PPARGC1A, that of PPARGC1B is not affected by cold exposure, obesity, fasting and exercise in brown adipose tissue, liver and muscle respectively [9]. Like the expression of PPARGC1A, that of the PPARGCIB isoform was shown to be reduced in type 2 diabetic patients and in their non-diabetic first-degree relatives, indicating that downregulation of both PPARGC1A and PPARGC1B is among the primary defects contributing to the development of

mitochondrial dysfunction, lipid accumulation, insulin resistance and ultimately type 2 diabetes.

However, other early detectable aberrations in the development of insulin resistance include high plasma NEFA levels and increased muscular lipid content [11]. Thus, the question remains whether the decreased level of the PPARGC1 isoforms (and mitochondrial dysfunction) is the cause of insulin resistance or the effect of the disturbed fatty acid metabolism that accompanies insulin resistance.

It is thus equally possible that frequent consumption of high-fat diabetogenic diets, which is associated with high plasma NEFA levels and/or muscular fat accumulation, leads to decreased PPARGCl expression with impaired mitochondrial function as a putative consequence. Therefore, we tested whether the acute elevation of plasma NEFA during a hyperinsulinaemic-euglycaemic clamp, leading to acute insulin resistance, was associated with downregulation of the expression of skeletal muscle genes involved in oxidative metabolism.

\section{Subjects, materials and methods}

\section{Subjects}

Nine healthy lean male volunteers without a family history of diabetes mellitus participated in the present study (age: $20.1 \pm 0.5$ years, BMI: $21.7 \pm 0.6 \mathrm{~kg} / \mathrm{m}^{2}$, body fat $16.0 \pm 1.3 \%$ ). The study protocol was reviewed and approved by the Medical Ethical Committee of Maastricht University and all subjects gave written informed consent before participating in the study.

\section{Experimental design}

All subjects underwent two euglycaemic-hyperinsulinaemic clamps with simultaneous infusion of glycerol or lipid in randomised order. Test days were separated by at least 1 week. On both experimental days, subjects arrived at the laboratory at $08.00 \mathrm{~h}$ after an overnight fast. Upon arrival at the laboratory, a percutaneous biopsy from the vastus lateralis muscle was obtained and fasting blood samples were taken. Subsequently, a 6-h euglycaemic-hyperinsulinaemic clamp with $40 \mathrm{mU} / \mathrm{m}^{2} / \mathrm{min}$ insulin (Actrapid; Novo Nordisk, Copenhagen, Denmark) and variable co-infusion of a $20 \%$ glucose solution to reach a blood glucose level of $\sim 5 \mathrm{mmol} / \mathrm{l}$ was started, combined with the infusion of either lipid or glycerol. Blood samples were taken at regular time points for determination of circulating NEFA.

In the lipid condition, subjects were co-infused with a $20 \%$ triacylglycerol emulsion (Intralipid; Fresenius Kabi, Bad Homburg, Germany) at the rate of $81 \mathrm{ml} / \mathrm{h}$ for $6 \mathrm{~h}$. As a control condition, subjects were co-infused with glycerol 
$(4.15 \mathrm{~g} / 100 \mathrm{ml})$ at the rate of $73 \mathrm{ml} / \mathrm{h}$ to match the infused amounts of glycerol in the lipid condition. After $6 \mathrm{~h}$ a second muscle biopsy was taken.

Blood analysis

Plasma NEFA concentrations were measured using the Wako NEFA C kit (Wako Chemicals, Neuss, Germany), with adjustments made to allow valid measurement of NEFA levels exceeding 1,000 $\mu \mathrm{mol} / 1$.

Intramuscular triacylglycerols

Fresh cryosections $(5 \mu \mathrm{m})$ were stained for intramuscular triacylglycerols (IMTG) by Oil Red O staining combined with immunolabelling of the basal membrane marker laminin to allow quantification of IMTG, as described previously [12].

Reverse transcription and quantitative PCR

Total RNA was extracted from skeletal muscle biopsies using Trizol reagent (Invitrogen, Breda, the Netherlands). One microgram of RNA was reverse-transcribed to cDNA using random hexamer primers and a Stratascript enzyme (Stratagene, Amsterdam, the Netherlands) and quantitative PCR was performed using a method similar to that described by Russell et al. [13]. To control for any variations due to efficiencies of the reverse transcription and PCR, acidic ribosomal phosphoprotein PO (36B4) was used as internal control. All PCR runs were performed in triplicate.

\section{Statistical analysis}

All values are expressed as mean \pm SEM. Changes in plasma NEFA were calculated as total area under the curve minus baseline values. To compare the effects of the different infusions between groups, a two-way repeated-measures ANOVA was performed. When significant differences were found, a paired Student's $t$-test with Bonferroni correction was used to determine pre- and post-experimental differences within conditions. Outcomes were regarded as statistically significant if $p<0.05$.

\section{Results}

Plasma NEFA and insulin sensitivity

Baseline plasma NEFA were similar in the two conditions (545 \pm 25 and $467 \pm 48 \mu \mathrm{mol} / 1$ in lipid and glycerol respectively; not significant). Lipid infusion increased plasma
NEFA levels $\sim 2.7$-fold $(p<0.001$ ), up to $1,475 \pm 88 \mu \mathrm{mol} / 1$ (Fig. 1a). In agreement with previous studies, the rise in plasma NEFA was accompanied by a decrease in wholebody insulin sensitivity, as manifested by a drop in glucose infusion rate $2-3 \mathrm{~h}$ after the start of the infusion. The changes in glucose infusion rate, calculated as the difference in glucose infusion rate between 120 and 150 and 330 and $360 \mathrm{~min}$, were significantly different between lipid and glycerol infusion $(p<0.001)$ and the reduction in insulin sensitivity within the lipid condition averaged $\sim 31 \%$ (Fig. $1 \mathrm{~b} ; p=0.001$ ).

The euglycaemic-hyperinsulinaemic clamp in itself (with glycerol as control) decreased plasma NEFA levels to $\sim 30 \%$ of baseline, to $129 \pm 14 \mu \mathrm{mol} / 1(p<0.001)$, and the change in plasma NEFA was significantly different between glycerol and lipid $(p<0.001)$.

Intramuscular triacylglycerols

The changes in IMTG levels were significantly different between lipid and glycerol infusion during the hyperinsulinaemic-euglycaemic clamp $(p=0.003)$. Lipid infusion resulted in a significant increase in IMTG (from 2.84 \pm 0.68 to $4.42 \pm 0.85 \%$; $p=0.02$; Fig. $1 \mathrm{c}$ ). During the hyperinsulinaemic-euglycaemic clamp plus glycerol infusion, IMTG tended to decrease (from $2.97 \pm 0.77$ to $1.56 \pm 0.57 \%$; $p=0.065$ ).

Skeletal muscle gene expression

The changes in expression levels of PPARGC1A and $P P A R G C 1 B$ were significantly different between lipid and glycerol infusion during a glucose clamp $(p=0.015$ and $p=0.026$ for PPARGC1A and PPARGC1B respectively). Lipid infusion downregulated the expression of PPARGC1A (Fig. $2 \mathrm{a}$ ) to $61 \%$ of basal values $(p=0.034)$, whereas there was a tendency for upregulation of PPARGC1A in the glycerol condition $(p=0.098)$. As was the case for $P P A R G C 1 A$, lipid infusion decreased the expression of PPARGC1B (Fig. 2b) down to $\sim 77 \%$ of preinfusion values $(p=0.039)$.

In addition, the changes in PPARA mRNA levels tended to be different between lipid infusion and the infusion of glycerol $(p=0.06)$. PPARA mRNA levels decreased significantly down to $52 \% \quad(p=0.025)$ of basal levels upon infusion of the lipid emulsion but remained unaltered upon glycerol infusion (Fig. 3). PPARD and PPARG remained unaffected by infusion of either lipid or glycerol (Table 1).

The change in gene expression of COX4I1 (cytochrome $c$ oxidase subunit IV isoform 1) (Fig. 4), the gene encoding a subunit of complex IV of the electron transport chain and transcriptionally regulated by PPARGC1, was not statistically different from that in the glycerol condition $(p=0.18)$, 


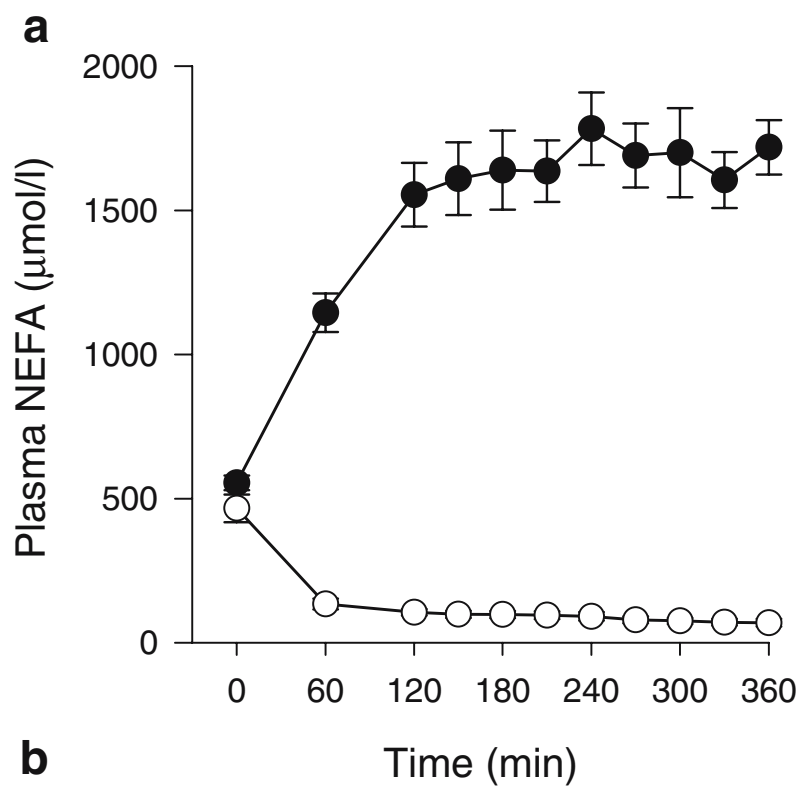

4 Fig. 1 Plasma NEFA levels (a) and glucose infusion rate (GIR) (b) during hyperinsulinaemic-euglycaemic clamp. (c) Intramuscular triacylglycerol (IMTG) levels before (pre) and after (post) the clamp procedure. The area fraction reflects the percentage of the total measured cell surface covered by lipid droplets. White bars/open circles, simultaneous glycerol infusion; black bars/closed circles, simultaneous lipid infusion. ${ }^{*} p<0.05$ compared with before infusion

although the expression of COX4II showed a greater decrease upon lipid infusion.

The expression of the gene for muscular carnitine palmitoyl transferase $1 \beta(C P T 1 B$, also known as $M$-CPT1), the rate-limiting enzyme in mitochondrial fatty acid uptake and subsequent oxidation, and the expression of the gene for acetyl-CoA carboxylase $\beta(A C A C B$, also known as $A C C 2$ ), which is involved in controlling fatty acid oxidation by inhibiting CPT1, were not affected by lipid infusion in comparison with glycerol (Table 1), although $C P T 1 B$ tended to be downregulated in the glycerol condition $(p=0.082)$.

The changes in mRNA levels of uncoupling protein 3 $(U C P 3)$, a protein involved in the mitochondrial handling of fatty acids, were significantly different between lipid and glycerol infusion $(p=0.003)$. UCP3 mRNA increased $\sim 1.8$ fold (Fig. $5 ; p=0.011$ ) upon lipid infusion, and tended to be downregulated in the glycerol condition $(p=0.066)$.

The gene expression of SOD2, the mitochondrial isoform of the endogenous anti-oxidant superoxide dismutase, was unchanged by either lipid or glycerol infusion (Table 1).

\section{Discussion}

The attractive hypothesis that subjects at risk of developing type 2 diabetes are susceptible to impaired mitochondrial biogenesis and functioning, which diminish fat oxidative capacity, is derived from the following consecutive observations: (1) older, insulin-resistant subjects displayed decreases in mitochondrial oxidative activity and ATP synthesis [14]; (2) similar decreases were observed in young insulin-resistant offspring of patients with type 2 diabetes [3]; (3) genes involved in mitochondrial oxidative phosphorylation and under the transcriptional control of PPARGC1A and PPARGC1B were coordinately downregulated in type 2 diabetes $[4,5]$; and (4) PPARGC1A and PPARGC1B themselves were also downregulated in humans with type 2 diabetes and, even more strikingly, in non-diabetic subjects with a family history of diabetes [4]. As a result of these impairments, nutrient excess, especially an increased supply of fatty acids to skeletal muscle, might further contribute to the deterioration of mitochondrial functioning and may promote intramuscular fat accumulation and muscle insulin resistance. However, it remains 

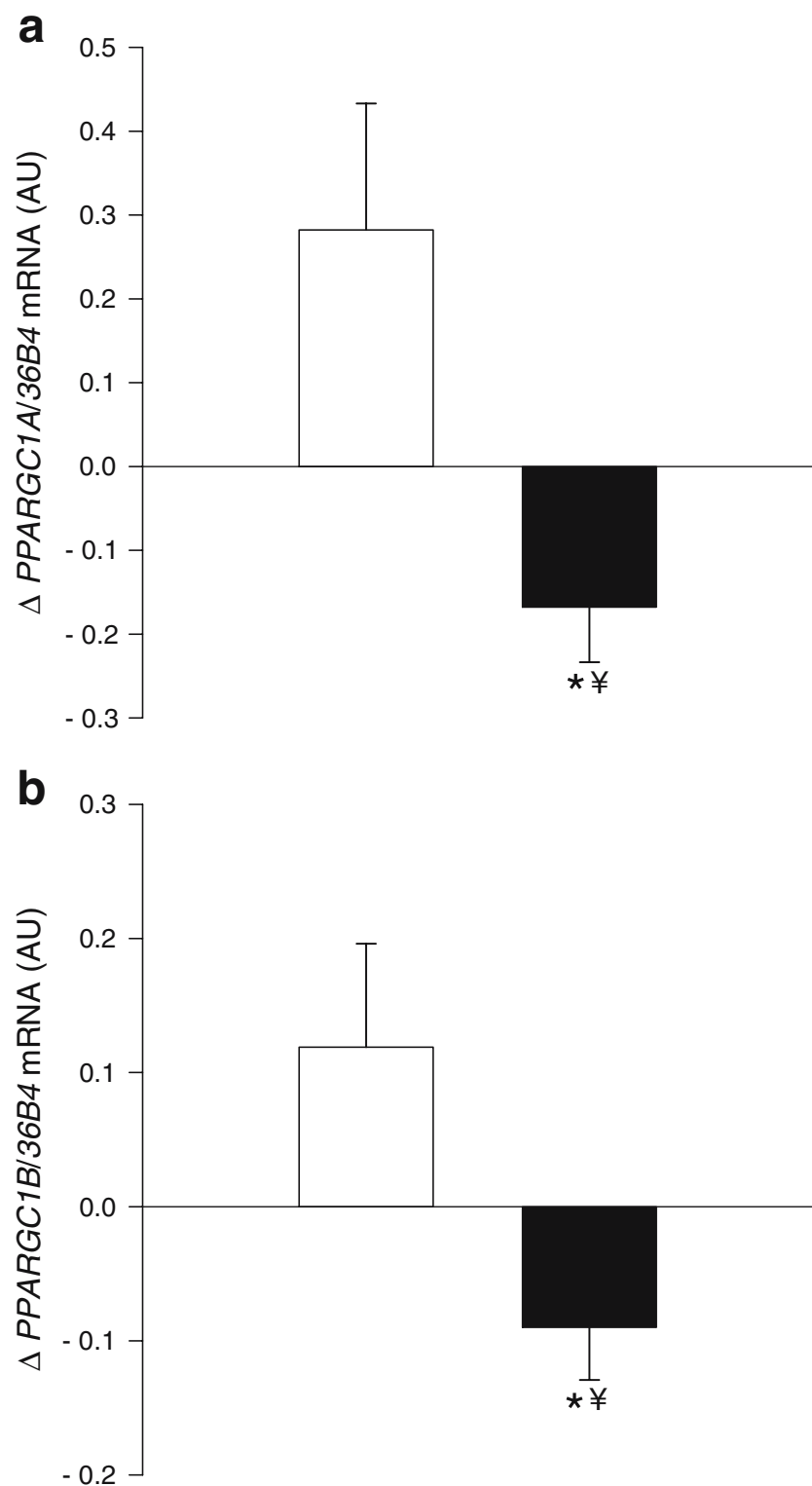

Fig. 2 Changes in gene expression of (a) $P P A R G C 1 A$ and (b) PPARGC1B in skeletal muscle after a 6-h hyperinsulinaemiceuglycaemic clamp with simultaneous infusion of glycerol (white bars) and lipid (black bars) ${ }^{*} p<0.05$ compared with baseline; ${ }^{*} p<0.05$ compared with glycerol

uncertain whether the disturbed mitochondrial functioning that was observed in the aetiology of type 2 diabetes is cause or effect with respect to intramuscular lipid accumulation.

Here, we show that an acute increase in the supply of longchain fatty acids, leading to insulin resistance and muscular fat accumulation, decreases the expression of PPARGC1A, PPARGC1B and PPARA mRNAs in skeletal muscle, indicating that acute elevation of NEFA levels, or resulting downstream effects, can interfere with the expression of these major activators of mitochondrial biogenesis and oxidative metabolism. The finding that PPARGC1A and, to a lesser extent, $P P A R G C 1 B$ tended to increase in the control condition fortifies the notion that PPARGCl homologues

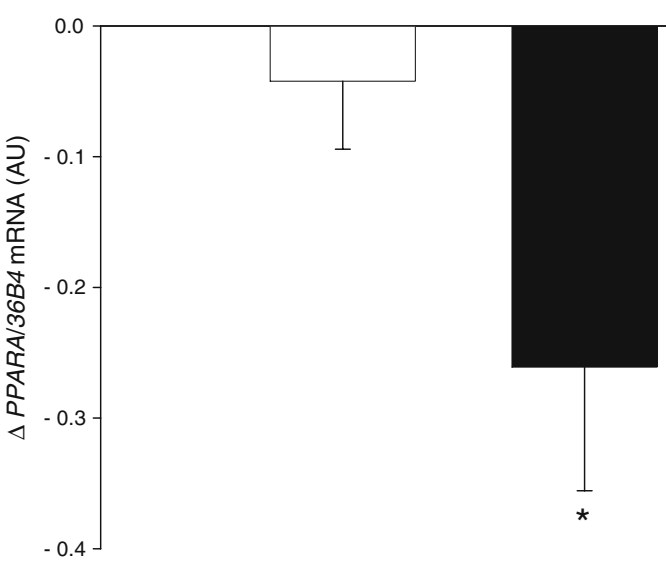

Fig. 3 Changes in gene expression of PPARA in skeletal muscle after a 6-h hyperinsulinaemic-euglycaemic clamp with simultaneous infusion of glycerol (white bar) and lipid (black bar) ${ }^{*} p<0.05$ compared with baseline

are downregulated upon lipid infusion and implies that the downregulation might even represent an underestimation of the lipid effect on PPARGCl expression. In the light of this, studies in cultured primary human skeletal myotubes revealed that insulin is indeed capable of inducing PPARGC1 expression [15].

Our results are in accordance with recent in vivo studies with comparable nutritional interventions showing a decrease in PPARGCl (isoform not specified) mRNA expression upon prolonged $(48 \mathrm{~h})$ lipid infusion [16] and a reduction in the expression of both PPARGC1A and PPARGC1B after a longer (3 days) dietary intervention of high-fat feeding in human subjects [17]. Although our results suggest that fatty acids might interfere directly with PPARGC1 transcription, results from in vitro studies on this topic are equivocal. Thus, human primary skeletal muscle myotubes treated with palmitate showed decreased expression of PPARGC1A in comparison with vehicle-treated myotubes [18], while Staiger et al. [19] showed a negative effect of palmitate on PPARGC1B but not on PPARGC1A in a similar cell model. The study of Staiger et al. further revealed that fatty acids with various degrees of saturation

Table 1 Skeletal muscle gene expression, expressed as a ratio with respect to the control gene $36 \mathrm{~B} 4$, before and after a hyperinsulinaemic-euglycaemic clamp with simultaneous infusion of glycerol and lipid

\begin{tabular}{llllll}
\hline Gene & \multicolumn{2}{l}{ Glycerol } & & & Lipid \\
\cline { 2 - 3 } & Before & After & & Before & After \\
\hline PPARB & $0.44 \pm 0.08$ & $0.57 \pm 0.13$ & & $0.50 \pm 0.10$ & $0.46 \pm 0.08$ \\
PPARG & $0.32 \pm 0.07$ & $0.25 \pm 0.1$ & & $0.26 \pm 0.06$ & $0.30 \pm 0.04$ \\
CPT1B & $0.62 \pm 0.07$ & $0.51 \pm 0.08$ & & $0.69 \pm 0.09$ & $0.59 \pm 0.07$ \\
ACACB & $0.30 \pm 0.03$ & $0.31 \pm 0.04$ & & $0.35 \pm 0.04$ & $0.40 \pm 0.07$ \\
SOD2 & $0.43 \pm 0.09$ & $0.55 \pm 0.06$ & & $0.40 \pm 0.09$ & $0.49 \pm 0.06$ \\
\hline
\end{tabular}




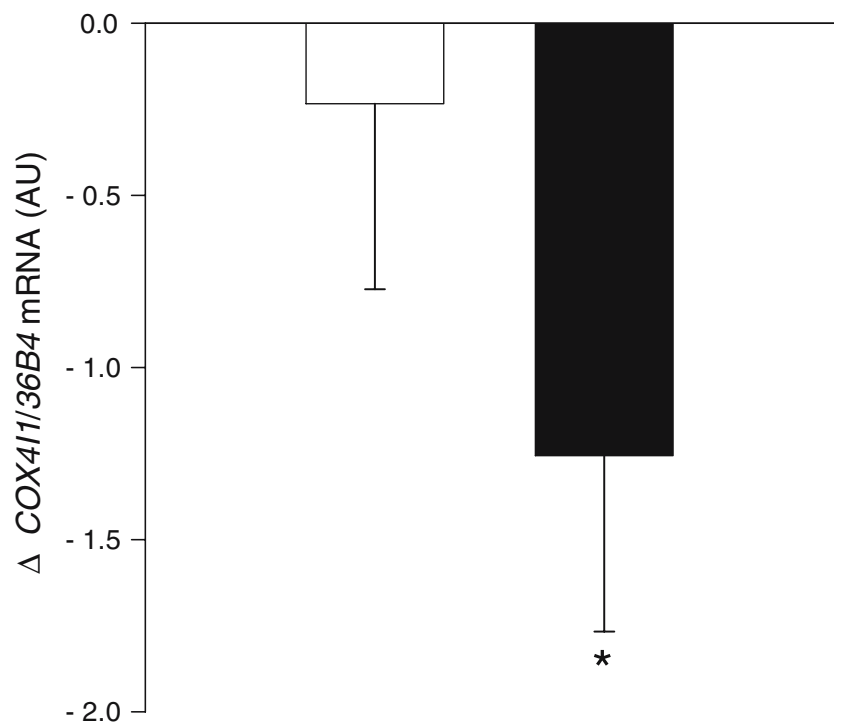

Fig. 4 Changes in skeletal muscle gene expression of $C O X 4 I 1$ after a 6-h hyperinsulinaemic-euglycaemic clamp with simultaneous infusion of glycerol (white bar) and lipid (black bar) ${ }^{*} p<0.05$ compared with baseline

had differential effects on the expression of the two $P P A R G C 1$ isoforms, indicating differences in transcriptional regulation between PPARGC1A and PPARGC1B.

Taken together, the results obtained in our acute lipidinduced insulin resistance model suggest that the downregulation of the PPARGC1 homologues observed in the (pre)diabetic state [5] may be the result of lipid-induced insulin resistance rather than being the cause. Since our 6-h lipid infusion also markedly increased the levels of

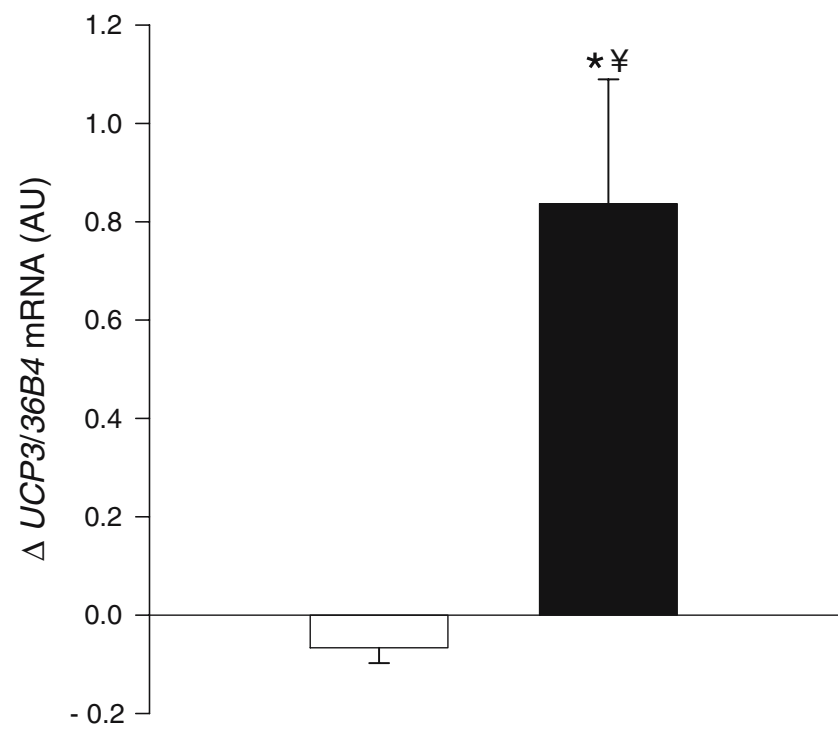

Fig. 5 Changes in gene expression of $U C P 3$ in skeletal muscle after a 6-h hyperinsulinaemic-euglycaemic clamp with simultaneous infusion of glycerol (white bar) and lipid (black bar) ${ }^{*} p<0.05$ compared with baseline; ${ }^{¥} p<0.05$ compared with glycerol intramuscular triacylglycerols, it remains possible that the active compounds affecting the expression of the PPARGC1 isoforms are not fatty acids in themselves, but metabolites originating from triacylglycerol formation. In this context it is interesting to note that an inverse relation has been reported between PPARGC1A protein levels and the synthesis rates of intramuscular triacylglycerols in rodent skeletal muscle [20].

To examine whether the downregulation of PPARGC1A and $P P A R G C 1 B$ had an effect on its downstream target genes involved in mitochondrial functioning, we measured the expression of cytochrome $c$ oxidase subunit 4 isoform 1 (COX4I1). PPARGC1A and PPARGC1B can increase the expression of nuclear respiratory factors $(N R F)$ and induce the coactivation of NRF1-mediated transcription of downstream target genes such as COX4I1 [21].

Although the time-frame of the present study, possibly in combination with limited statistical power, did not reveal a significant difference in the change in COX4II expression between the glycerol and lipid conditions, the reduction in COX4I1 seemed more pronounced upon lipid infusion. The long-term experiment by Sparks et al. [17] did show decreased levels of genes involved in oxidative phosphorylation along with the decreased levels of PPARGC1A and PPARGC1B, suggesting that the time-frame of the present study was too short to display obvious effects downstream of PPARGC1.

To further examine whether lipid-induced insulin resistance also affected other key genes involved in oxidative metabolism, we measured the expression of $C P T 1 B$ and $A C A C B$, which encode key regulators of fat oxidation. We found that lipid infusion did not affect $C P T 1 B$ and $A C A C B$ expression within the time frame of the present study.

In addition to reduced expression of oxidative genes, possibly explained by the accumulation of fat in skeletal muscle, patients with type 2 diabetes are also characterised by increased mitochondrial damage [22]. In our laboratory we have previously hypothesised that the accumulation of fat in the vicinity of muscle mitochondria may lead to reactive oxygen species-induced lipid peroxidation and that the lipid peroxides produced may be responsible for the damage to mitochondrial proteins and structure [23]. Indeed, it has recently been shown that insulin-resistant subjects display increased levels of lipid peroxides [24]. In addition, we and others have hypothesised a role for uncoupling protein 3 (UCP3) in the protection of mitochondria against this lipid-induced damage, either by directly diminishing the production of reactive oxygen species $[25,26]$ or by exporting lipid peroxides [27] or fatty acid anions [28] away from the mitochondria. In accordance with the increased level of lipid peroxides and mitochondrial damage, patients with type 2 diabetes were indeed shown to have a $50 \%$ reduction in UCP3 protein levels [29]. The increased expression of UCP3 upon lipid 
infusion (which increases IMTG) supports the idea that UCP3 protects against the accumulation of detrimental lipids, irrespective of the exact mechanism by which UCP3 can do so. Interestingly, Vidal et al. [30] showed that the fatty acid-induced induction of UCP 3 mRNA expression was impaired in patients with type 2 diabetes, suggesting an impairment of the defence mechanism that protects mitochondria against lipid-induced damage.

Another way to reduce lipid-induced oxidative damage is by increasing mitochondrial antioxidant capacity. Therefore, we measured gene expression of SOD2 but found no effect of lipid infusion on the expression levels of this gene. This suggests that UCP3 may be specifically involved in the defence against lipid-induced mitochondrial damage, although gene expression of SOD2 and UCP3 does not necessarily reflect protein levels, let alone the biological activity of the proteins.

In summary, we showed in the present study that expression of PPARGC1A, PPARGC1B and PPARA in skeletal muscle was decreased upon a $6 \mathrm{~h}$ infusion of long-chain triacylglycerols that lead to muscular fat accumulation and insulin resistance. In addition, UCP3 expression was upregulated upon lipid infusion, which is in accordance with the putative role of UCP3 in the protection of mitochondria against lipotoxicity. Although it should be noted that changes in gene expression do not necessarily reflect changes in the levels of functional proteins, the downregulation of PPARA expression upon lipid infusion, in addition to the downregulation of PPARGCl, could have a detrimental effect on mitochondrial biogenesis and functioning upon chronic exposure to elevated plasma NEFA levels. This may explain the downregulation of oxidative genes in type 2 diabetes mellitus.

Acknowledgements This study was funded by the Wageningen Centre for Food Sciences, an alliance of major Dutch food industries, TNO Nutrition and Food Research and Wageningen University and Research Centre, with financial support from the Dutch government. J. Hoeks was supported by a grant from the Netherlands Organization for Scientific Research (NWO) and the research of P. Schrauwen was made possible by a fellowship of the Royal Netherlands Academy of Arts and Sciences. M. K. C. Hesselink is supported by a VIDI Research Grant for innovative research from the Netherlands Organization for Scientific Research (Grant 917.66.359).

Duality of interest The authors declare that no duality of interest exists.

\section{References}

1. Krssak M, Falk Petersen K, Dresner A et al (1999) Intramyocellular lipid concentrations are correlated with insulin sensitivity in humans: a $1 \mathrm{H}$ NMR spectroscopy study. Diabetologia 42:113-116

2. Itani SI, Ruderman NB, Schmieder F, Boden G (2002) Lipidinduced insulin resistance in human muscle is associated with changes in diacylglycerol, protein kinase C, and IkappaB-alpha. Diabetes 51:2005-2011

3. Petersen KF, Dufour S, Befroy D, Garcia R, Shulman GI (2004) Impaired mitochondrial activity in the insulin-resistant offspring of patients with type 2 diabetes. N Engl J Med 350:664-671

4. Mootha VK, Lindgren CM, Eriksson KF et al (2003) PGC-1alpharesponsive genes involved in oxidative phosphorylation are coordinately downregulated in human diabetes. Nat Genet 34:267-273

5. Patti ME, Butte AJ, Crunkhorn S et al (2003) Coordinated reduction of genes of oxidative metabolism in humans with insulin resistance and diabetes: potential role of PGC1 and NRF1. Proc Natl Acad Sci USA 100:8466-8471

6. Lin J, Wu H, Tarr PT et al (2002) Transcriptional co-activator PGC-1 alpha drives the formation of slow-twitch muscle fibres. Nature 418:797-801

7. Lin J, Tarr PT, Yang R et al (2003) PGC-1beta in the regulation of hepatic glucose and energy metabolism. J Biol Chem 278:30843-30848

8. St-Pierre J, Lin J, Krauss S et al (2003) Bioenergetic analysis of peroxisome proliferator-activated receptor gamma coactivators 1alpha and 1beta (PGC-1alpha and PGC-1beta) in muscle cells. J Biol Chem 278:26597-26603

9. Meirhaeghe A, Crowley V, Lenaghan C et al (2003) Characterization of the human, mouse and rat PGC1 beta (peroxisomeproliferator-activated receptor-gamma co-activator 1 beta) gene in vitro and in vivo. Biochem J 373:155-165

10. Kamei Y, Ohizumi H, Fujitani Y et al (2003) PPARgamma coactivator 1 beta/ERR ligand 1 is an ERR protein ligand, whose expression induces a high-energy expenditure and antagonizes obesity. Proc Natl Acad Sci USA 100:12378-12383

11. Jacob S, Machann J, Rett K et al (1999) Association of increased intramyocellular lipid content with insulin resistance in lean nondiabetic offspring of type 2 diabetic subjects. Diabetes 48:1113-1119

12. Roorda BD, Hesselink MK, Schaart G et al (2005) DGAT1 overexpression in muscle by in vivo DNA electroporation increases intramyocellular lipid content. J Lipid Res 46:230-236

13. Russell AP, Hesselink MK, Lo SK, Schrauwen P (2005) Regulation of metabolic transcriptional co-activators and transcription factors with acute exercise. FASEB J 19:986-988

14. Petersen KF, Befroy D, Dufour S et al (2003) Mitochondrial dysfunction in the elderly: possible role in insulin resistance. Science 300:1140-1142

15. Al-Khalili L, Forsgren M, Kannisto K, Zierath JR, Lonnqvist F, Krook A (2005) Enhanced insulin-stimulated glycogen synthesis in response to insulin, metformin or rosiglitazone is associated with increased mRNA expression of GLUT4 and peroxisomal proliferator activator receptor gamma co-activator 1. Diabetologia 48:1173-1179

16. Richardson DK, Kashyap S, Bajaj M et al (2005) Lipid infusion decreases the expression of nuclear encoded mitochondrial genes and increases the expression of extracellular matrix genes in human skeletal muscle. J Biol Chem 280:10290-10297

17. Sparks LM, Xie H, Koza RA et al (2005) A high-fat diet coordinately downregulates genes required for mitochondrial oxidative phosphorylation in skeletal muscle. Diabetes 54:1926-1933

18. Southgate RJ, Bruce CR, Carey AL et al (2005) PGC-1alpha gene expression is down-regulated by Akt-mediated phosphorylation and nuclear exclusion of FoxO1 in insulin-stimulated skeletal muscle. FASEB J 19:2072-2074

19. Staiger H, Staiger K, Haas C, Weisser M, Machicao F, Haring HU (2005) Fatty acid-induced differential regulation of the genes encoding peroxisome proliferator-activated receptor-gamma coactivator-1alpha and -1beta in human skeletal muscle cells that have been differentiated in vitro. Diabetologia 48:2115-2118

20. Benton CR, Han XX, Febbraio M, Graham TE, Bonen A (2006) Inverse relationship between PGC-1alpha protein expression and 
triacylglycerol accumulation in rodent skeletal muscle. J Appl Physiol 100:377-383

21. Wu Z, Puigserver P, Andersson U et al (1999) Mechanisms controlling mitochondrial biogenesis and respiration through the thermogenic coactivator PGC-1. Cell 98:115-124

22. Kelley DE, He J, Menshikova EV, Ritov VB (2002) Dysfunction of mitochondria in human skeletal muscle in type 2 diabetes. Diabetes 51:2944-2950

23. Schrauwen P, Hesselink MK (2004) Oxidative capacity, lipotoxicity, and mitochondrial damage in type 2 diabetes. Diabetes 53:1412-1417

24. Russell AP, Gastaldi G, Bobbioni-Harsch E et al (2003) Lipid peroxidation in skeletal muscle of obese as compared with endurance-trained humans: a case of good vs. bad lipids? FEBS Lett 551:104-106

25. Echtay KS, Esteves TC, Pakay JL et al (2003) A signalling role for 4-hydroxy-2-nonenal in regulation of mitochondrial uncoupling. EMBO J 22:4103-4110
26. Brand MD, Buckingham JA, Esteves TC et al (2004) Mitochondrial superoxide and aging: uncoupling-protein activity and superoxide production. Biochem Soc Symp (71):203-213

27. Goglia F, Skulachev VP (2003) A function for novel uncoupling proteins: antioxidant defense of mitochondrial matrix by translocating fatty acid peroxides from the inner to the outer membrane leaflet. FASEB J 17:1585-1591

28. Schrauwen P, Hoeks J, Schaart G et al (2003) Uncoupling protein 3 as a mitochondrial fatty acid anion exporter. FASEB J 17:22722274

29. Schrauwen P, Hesselink MK, Blaak EE et al (2001) Uncoupling protein 3 content is decreased in skeletal muscle of patients with type 2 diabetes. Diabetes 50:2870-2873

30. Vidal H, Langin D, Andreelli F, Millet L, Larrouy D, Laville M (1999) Lack of skeletal muscle uncoupling protein 2 and 3 mRNA induction during fasting in type-2 diabetic subjects. Am J Physiol 277:E830-E837 